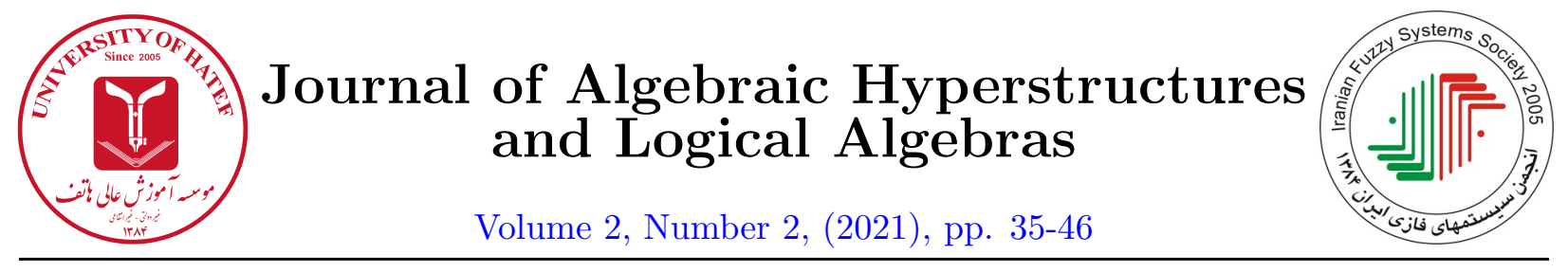

\title{
On $\alpha$-solvable fundamental groups
}

\author{
F. Mohammadzadeh ${ }^{1}$ and E. Mohammadzadeh ${ }^{2}$ \\ ${ }^{1,2}$ Department of Mathematics, Faculty of Science, Payame Noor University, 19395-3697, Tehran, Iran \\ mohamadzadef464@gmail.com, mohamadzadeh36@gmail.com
}

\begin{abstract}
We introduce a specific kind of equivalence relation $\xi_{n}^{* \alpha}$ on a fuzzy hypergroup $S$ such that the quotient $S / \xi^{* \alpha}$, the set of all equivalence classes, is an $\alpha$-solvable group. This helps us to introduce the $\alpha$-solvable fundamental relation $\xi^{* \alpha}$. In particular, we obtain an equivalent condition with transitivity of $\xi^{\alpha}$.
\end{abstract}

\section{Article Information}

Corresponding Author:

F. Mohammadzadeh;

Received: April 2021;

Revised: May 2021;

Accepted: June 2021;

Paper type: Original.

\section{Keywords:}

Fuzzy hypergroup, fundamental relation, $\alpha$-solvable group.

\section{Introduction}

A solvable group with respect to an automorphism $\alpha$ is called an $\alpha$-solvable group. An $\alpha$-solvable group is a group that $\alpha$-derived series terminates in the trivial subgroups. In [2], $\alpha$-solvable groups, as a generalization of solvable groups, were introduced and some properties of $\alpha$-solvabel groups were discussed. Clearly, every solvable group is an $\alpha$-solvable group, where $\alpha$ is the identity automorphism.

In 1965, Zadeh [14] proposed the concept of fuzzy sets. In 1971, Rosenfeld [12], applied fuzzy sets in group theory to introduce fuzzy subgroups of a group. Fuzzy hypergroups as a new approach on fuzzy sets, introduced by Corsini and Tofan [5]. The basic idea is that a fuzzy hyperoperation assigns to every pair of elements a fuzzy set. Some researchers extended the concepts of abstract

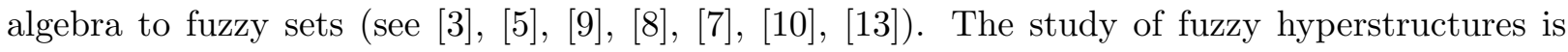
an interesting topic on fuzzy sets theory. One way for connecting fuzzy hypergroups and groups is the fundamental relation. A fundamental relation of a hypergroup is the smallest equivalence

https://doi.org/10.52547/HATEF.JAHLA.2.2.35 
relation such that a quotient is a group. The fundamental relation, $\beta$, as a vital concept on hyperstructures, is studied by many scholars [4]. This relation plays an important role in the theory of hyperstructures. Also, the relation $\gamma^{*}$ is the least equivalence relation on a hypergroup $H$ such that a quotient is an abelian group [6]. Moreover, $\gamma^{*}$ is a commutative fundamental relation. It is known that if $R$ is a fuzzy strongly regular equivalence relation on a fuzzy hypergroup $S$, then we can define a binary operation $\otimes$ on the quotient set $S / R$, the set of all equivalence classes of $S$ with respect to $R$, such that $(S / R, \otimes)$ is a group (see [I.3]). Ameri and Nozari [U], followed the results obtained by Sen et. all on fuzzy hypersemigroups to introduce the fundamental relation of fuzzy hypersemigroups. Now, we introduce an $\alpha$-solvable fundamental group. In addition, we define a strongly regular relation $\xi^{* \alpha}$ on a fuzzy hypergroup $S$. Then we prove that $S / \xi^{* \alpha}$, the set of all equivalence classes of $\xi^{* \alpha}$ under usual operation, is an $\alpha$-solvable group. Finally, by the notion of $\xi^{* \alpha}$-part of a fuzzy hypergroup, we try to get an equivalent condition to transitivity of $\xi^{* \alpha}$.

\section{Preliminaries}

Let $G$ be any group and $\alpha$ be an automorphism of $G$. For two elements $x$ and $y$ of $G$ the $\alpha$ commutator of $G$ is $[x, y]_{\alpha}=x y x^{-1} y^{-\alpha}$, where $y^{-\alpha}$ is used for $\alpha\left(y^{-1}\right)$. For any $x_{1}, x_{2}, \ldots, x_{n}$ of $G$ one can define inductively $\left[x_{1}, x_{2}, \ldots, x_{n}\right]_{\alpha}$, the $\alpha$-commutator of weight $n$, as follows:

$$
\left[x_{1}, x_{2}, \ldots, x_{n}\right]_{\alpha}=\left[x_{1},\left[x_{2}, \ldots, x_{n}\right]_{\alpha}\right]_{\alpha} .
$$

For any non-empty subsets $X_{1}$ and $X_{2}$ of $G$ the $\alpha$-commutator subgroup of $G$, denoted by $\left[X_{1}, X_{2}\right]_{\alpha}$ is defined as the subgroup of $G$ generated by the set $\left\{\left[x_{1}, x_{2}\right]_{\alpha} \mid x_{1} \in X_{1}, x_{2} \in X_{2}\right\}$. It is clear that $\left[X_{1}, X_{2}\right]_{\alpha}$ is not equal to $\left[X_{2}, X_{1}\right]_{\alpha}$ in general. Let $N$ be a normal subgroup of $G$ and $N^{\alpha}=N$. For the isomorphism $\bar{\alpha}: G / N \longrightarrow G / N$ given by $\bar{x}^{\bar{\alpha}}=x^{\alpha} N$ we have $\left[\overline{x_{1}}, \overline{x_{2}}, \ldots, \overline{x_{n}}\right]_{\bar{\alpha}}=$ $\left[x_{1}, x_{2}, \ldots, x_{n}\right]_{\alpha} N$ (see [2]).

The $\alpha$-derived subgroup of a group $G$ with respect to an automorphism $\alpha$ is defined by $D^{\alpha}(G)=$ $\left\langle[x, y]_{\alpha} \mid x, y \in G\right\rangle$. Also, $D_{0}^{\alpha}(G)=G, D_{1}^{\alpha}(G)=D^{\alpha}(G)$ and $D_{i}^{\alpha}(G)=D^{\alpha}\left(D_{i-1}^{\alpha}(G)\right)$. A group $G$ is $\alpha$-solvable if and only if for some integer $r, D_{r}^{\alpha}(G)=\{1\}$, where 1 is the identity element. The smallest such $r$ is called length of $G$ (see [2]).

A hypergroupoid is a nonempty set $H$ with a hyperoperation $\triangleright$ defined on $H$, that is, a mapping of $H \times H$ into the family of non-empty subsets of $H$. If $(x, y) \in H \times H$, then its image under $\triangleright$ is denoted by $x \triangleright y$. If $A, B$ are non-empty subsets of $H$, then $A \triangleright B$ is given by $A \triangleright B=\bigcup\{x \triangleright y \mid x \in$ $A, y \in B\}$. Thus $x \triangleright A$ is used for $\{x\} \triangleright A$ and $A \triangleright x$ for $A \triangleright\{x\}$. Generally, the singleton $a$ is identified with its member $a$. The structure $(H, \triangleright)$ is called a semihypergroup if $a \triangleright(b \triangleright c)=(a \triangleright b) \triangleright c$ for any $a, b, c \in H$, and a semihypergroup $(H, \triangleright)$ is called a hypergroup in the sense of Marty if $x \triangleright H=H \triangleright x=H$, for any $x \in H$. This axiom means that for any $x, y \in H$ there exist $u, v \in H$ such that $y \in x \triangleright u$ and $y \in v \triangleright x$.

Let $S$ be a non-empty set and $F^{*}(S)$ be the set of all non-zero fuzzy subsets of $S$. We denote by 0 the zero fuzzy set. Then $\circ: S \times S \rightarrow F^{*}(S)$ is a fuzzy hyperoperation on $S$ and the couple $(S, \circ)$ is called a fuzzy hypergroupoid.

Let $\mu, \nu$ be two fuzzy subsets of a fuzzy hypergroupoid $(S, \circ)$. In [13] for any $a, r \in S$ we have the following statements:

(i) $(\mu \odot \nu)(r)=\bigvee_{p, q \in S}(\mu(p) \wedge(p \circ q)(r) \wedge \nu(q))$, 
(ii) $(a \bullet \mu)(r)= \begin{cases}\bigvee_{t \in S}((a \circ t)(r) \wedge \mu(t)), & \mu \neq 0 \\ 0 . & \mu=0,\end{cases}$

$$
(\mu \bullet a)(r)= \begin{cases}\bigvee_{t \in S}(\mu(t) \wedge(t \circ a)(r)), & \mu \neq 0 \\ 0 . & \mu=0\end{cases}
$$

Definition 2.1. [1]3]

(i) A fuzzy hypergroupoid $(S, \circ)$ is a fuzzy semihypergroup if for any $x, y, z \in S$ we have $(x \circ y) \bullet z=$ $x \bullet(y \circ z)$.

(iii) A fuzzy semihypergroup is a fuzzy hypergroup (FHG) if $x \circ S=\chi_{S}=S \circ x$. A fuzzy subhypergroup $(K, \circ)$ of an $F H G(S, \circ)$ is a non-empty subset $K \subseteq S$ such that for any $k \in K$, $k \circ K=K \circ k=\chi_{K}$. Let $\left(S_{1}, \circ_{1}\right)$ and $\left(S_{2}, \circ_{2}\right)$ be two FHG. A map $f: S_{1} \rightarrow S_{2}$ is called a fuzzy hypergroup homomorphism if, for any $x, y \in S_{1}, f\left(x \circ_{1} y\right)=f(x) \circ_{2} f(y)$.

Definition 2.2. [13] Let $\rho$ be an equivalence relation on a fuzzy semihypergroup $(S, \circ)$ and $\mu, \nu$ be two fuzzy subsets of $(S, \circ)$. We say that $\mu \overline{\bar{\rho}} \nu$ if for all $x, y \in S$ such that $\mu(x)>0$ and $\nu(y)>0$, then $x \rho y$.

Definition 2.3. [13] An equivalence relation $\rho$ on a fuzzy semihypergroup $(S, \circ)$ is said to be a fuzzy strongly regular relation if $a \rho b$ and $a^{\prime} \rho b^{\prime}$ imply $a \circ a^{\prime} \overline{\bar{\rho}} b \circ b^{\prime}$.

Theorem 2.4. [1.3] Let $(S, \circ)$ be a fuzzy semihypergroup and $\rho$ be an equivalence relation on $S$. For any $\rho_{a}, \rho_{b} \in S / \rho$ consider the operation $\oplus$ as follows:

$$
\rho_{a} \oplus \rho_{b}=\left\{\rho_{c} \mid\left(a^{\prime} \circ b^{\prime}\right)(c)>0, a \rho a^{\prime}, b \rho b^{\prime}\right\} .
$$

Then $\rho$ is a fuzzy strongly regular relation on $(S, \circ)$ iff $(S / \rho, \oplus)$ is a semigroup.

Definition 2.5. [I, П]] Let $(S, \circ)$ be a fuzzy semihypergroup and $\mathbb{S}_{n}$ be the symmetric group on $n$ letters $(n \in \mathbb{N})$. We define the relations $\lambda$ and $\epsilon_{n}$ on $S$ in the following way:

$$
a \lambda b \Leftrightarrow \exists x_{1}, \ldots, x_{n} \in S, \text { such that }\left(x_{1} \circ \ldots \circ x_{n}\right)(a)>0 \quad \text { and }\left(x_{1} \circ \ldots \circ x_{n}\right)(b)>0 .
$$

(ii) $\epsilon=\bigcup_{n \geq 1} \epsilon_{n}$, where $\epsilon_{1}=\{(s, s) \mid s \in S\}$ and for any $n \geq 2$;

$$
a \epsilon_{n} b \Leftrightarrow \exists x_{1}, \ldots, x_{n} \in S, \exists \sigma \in \mathbb{S}_{n} \text { such that }\left(x_{1} \circ \ldots \circ x_{n}\right)(a)>0 \text { and }\left(x_{\sigma_{1}} \circ \ldots \circ x_{\sigma_{n}}\right)(b)>0 .
$$

One can see that $\lambda$ and $\epsilon$ are symmetric and reflexive. Let $\epsilon^{*}$ and $\lambda^{*}$ be the transitive closure of $\epsilon$ and $\lambda$, respectively. Then $\epsilon^{*}$ and $\lambda^{*}$ are equivalence relations.

Definition 2.6. [13] Let $(S, \circ)$ be a fuzzy semihypergroup. The smallest equivalence relation $\rho$ on $S$ is called the fundamental relation if the quotient structure $(S / \rho, \oplus)$ is a semigroup.

Theorem 2.7. [I] The relation $\epsilon^{*}$ is the abelian fundamental relation on fuzzy semihypergroup $(S, \circ)$.

\section{Characterization of $\alpha$-solvable groups via a fuzzy strongly reg- ular relation}

We introduce a new fuzzy strongly regular relation on an FHG such that the quotient group is an $\alpha$-solvable group.

Note: Let $(S, \circ)$ be an FHG and $m \in \mathbb{N}$. From now on for simplify we use the following notations: 
(1)For any $x, y \in S$ we use $x y$ instead of $x \circ y$.

(2) For any fuzzy strongly regular relation $\rho$ on $S$ and any $x \in X$ we use $\bar{x}$ for $\rho_{x}$.

(3) For any $z_{1}, \ldots, z_{m}$ of $S$ we denote $z_{1} \circ z_{2}, \cdots \circ z_{m}$ by $\prod_{i=1}^{m} z_{i}$.

(4) Let $\operatorname{Aut}(S)$ denote the set of all one to one and onto fuzzy homomorphisms on an FHG.

Definition 3.1. Let $(S, \circ)$ be an FHG and $\alpha \in A u t(S)$. Suppose $A_{0}^{\alpha}(S)=S$ and for any $k \geq 0$, $A_{k+1}^{\alpha}(S)=\left\{t \in S \mid \exists r \in S\right.$ such that $(x y)(r)>0$ and $\left(t \bullet y^{\alpha} x\right)(r)>0$ for some $\left.x, y \in A_{k}^{\alpha}(S)\right\}$.

For integers $n \geq 1$ and $m>1$, consider $\xi_{1, n}^{\alpha}$ is the diagonal relation on $S$. We define the relation $\xi_{m, n}^{\alpha}$ as follows:

$x \xi_{m, n}^{\alpha} y \Leftrightarrow \exists\left(z_{1}, \ldots, z_{m}\right) \in S^{m}, \exists \sigma \in \mathbb{S}_{m}$ with $\sigma(i)=i$ if $z_{i} \notin A_{n}^{\alpha}(S)$ such that

$$
\left(\prod_{i=1}^{m} z_{i}\right)(x)>0 \text { and }\left(\prod_{i=1}^{m} z_{\sigma(i)}\right)(y)>0
$$

Consider $\xi_{n}^{\alpha}=\bigcup_{m \geq 1} \xi_{m, n}^{\alpha}$. Then $\xi_{n}^{* \alpha}$, the transitive closure of $\xi_{n}^{\alpha}$, is an equivalence relation on $S$, since $\xi_{n}^{\alpha}$ is symmetric. For this let $a \xi_{n}^{\alpha} b$. Then there exists an integer $m \geq 1$ such that $a \xi_{m, n}^{\alpha} b$. It follows that

$\exists\left(z_{1}, \ldots, z_{m}\right) \in S^{m}, \exists \delta \in \mathbb{S}_{m}$ with $\delta(i)=i$ if $z_{i} \notin A_{n}^{\alpha}(S)$ such that $\left(\prod_{i=1}^{m} z_{i}\right)(a)>0$ and $\left(\prod_{i=1}^{m} z_{\delta(i)}\right)(b)>0$.

Put $I=\delta(i)$. Now, for $\left(z_{1}, \ldots, z_{m}\right) \in S^{m}$ and $\delta^{-1} \in \mathbb{S}_{m}$ with $\delta^{-1}(I)=i$ if $z_{I} \notin A_{n}^{\alpha}(S)$, then $\left(\prod_{I=1}^{m} z_{I}\right)(a)>0$ and $\left(\prod_{I=1}^{m} z_{\delta(I)}\right)(b)>0$. Therefore, $b \xi_{n}^{\alpha} a$ and so $\xi_{n}^{\alpha}$ is symmetric. Also, $\xi_{n}^{\alpha}$ is reflexive. Since for any $a \in S$ we have $a(a)=\left(\chi_{a}\right)(a)=1$.

Example 3.2. Let $S=\mathbb{Z}_{2}$ and $\alpha$ be the identity isomorphism. For any $x, y \in \mathbb{Z}_{2}$ we define a fuzzy hyper operation $\circ$ on $\mathbb{Z}_{2}$ by $x \circ y=\chi_{\{x, y\}}$. Clearly, $\left(\mathbb{Z}_{2}, \circ\right)$ is an $F H G$ and $A_{0}^{\alpha}\left(\mathbb{Z}_{2}\right)=\mathbb{Z}_{2}$. Also,

$$
A_{1}^{\alpha}\left(\mathbb{Z}_{2}\right)=\left\{t \in \mathbb{Z}_{2} \mid \exists r \in \mathbb{Z}_{2} ;(x \circ y)(r)>0 \text { and }\left(t \bullet\left(y^{\alpha} \circ x\right)\right)(r)>0 \text {, for some } x, y \in \mathbb{Z}_{2}\right\} \text {. }
$$

Let $r=0, x=0$ and $y=1$. Then $(x \circ y)(r)=\chi_{\{x, y\}}(r)=\chi_{\{0,1\}}(0)>0$ and

$$
\begin{aligned}
\left(t \bullet\left(y^{\alpha} \circ x\right)\right)(r) & =\bigvee_{s \in \mathbb{Z}_{2}}(t \circ s)(r) \wedge\left(y^{\alpha} \circ x\right)(s) \\
& =\bigvee_{s \in \mathbb{Z}_{2}} \chi_{\{t, s\}}(0) \wedge \chi_{\left\{0,1^{\alpha}\right\}}(s) \\
& =\left(\chi_{\{t, 0\}}(0) \wedge \chi_{\{1,0\}}(0)\right) \vee\left(\chi_{\{t, 1\}}(0) \wedge \chi_{\{1,0\}}(1)\right) \\
& =1 .
\end{aligned}
$$

Therefore, $A_{1}^{\alpha}\left(\mathbb{Z}_{2}\right)=\mathbb{Z}_{2}$. 
Example 3.3. Let $\alpha$ be the identity isomorphism and $S=\{a, b, c\}$. We define the fuzzy hyperoperation " ○" on $S$ as follows:

$(a \circ a)(a)=(b \circ b)(a)=(c \circ c)(a)=0.5,(a \circ b)(b)=(b \circ a)(b)=(b \circ c)(b)=(c \circ b)(b)=$ $0.1,(a \circ c)(c)=(b \circ b)(c)=(c \circ a)(c)=0.7$, and $(a \circ a)(b)=(a \circ a)(c)=(a \circ b)(a)=(a \circ b)(c)=$ $(a \circ c)(a)=(a \circ c)(b)=(b \circ a)(a)=(b \circ a)(c)=(b \circ b)(b)=(b \circ c)(a)=(b \circ c)(c)=(c \circ a)(a)=$ $(c \circ a)(b)=(c \circ b)(a)=(c \circ b)(c)=(c \circ c)(b)=(c \circ c)(c)=0$.

Let $\rho=\{(a, a),(b, b),(c, c),(a, c),(c, a)\}$. Then $A_{0}^{\alpha}(S)=S$ and $A_{1}^{\alpha}(S)=\{a\}$.

Theorem 3.4. The relation $\xi_{n}^{* \alpha}$ is a fuzzy strongly regular relation.

Proof. It is clear that $\xi_{m, n}^{* \alpha}$ is an equivalence relation. First, we show that for any $x, y, z \in S$

$$
x \xi_{n}^{\alpha} y \Rightarrow x z \overline{\overline{\xi_{n}^{\alpha}}} y z \text { and } z x \overline{\overline{\xi_{n}^{\alpha}}} z y
$$

If $x \xi_{n}^{\alpha} y$, then there exists an integer $m$ such that $x \xi_{m, n}^{\alpha} y$, and so there exist $\left(z_{1}, \ldots, z_{m}\right) \in S^{m}$ and $\sigma \in \mathbb{S}_{m}$ with $\sigma(i)=i$ if $z_{i} \notin A_{n}^{\alpha}(S)$ such that $\left(\prod_{i=1}^{m} z_{i}\right)(x)>0$ and $\left(\prod_{i=1}^{m} z_{\sigma(i)}\right)(y)>0$.

Let $z \in S$ such that for any $r, s$ we have $(x z)(r)>0$ and $(y z)(s)>0$. Let $p=x$ and $q=y$. Then

$$
\left(\left(\prod_{i=1}^{m} z_{i}\right) \bullet z\right)(r)=\bigvee_{p}\left\{\left(\prod_{i=1}^{m} z_{i}\right)(p) \wedge(p z)(r)\right\}>0
$$

and

$$
\left(\left(\prod_{i=1}^{m} z_{\sigma(i)}\right) \bullet z\right)(s)=\bigvee_{q}\left\{\left(\prod_{i=1}^{m} z_{\sigma(i)}\right)(q) \wedge(q z)(s)\right\}>0 .
$$

Now, suppose that $z_{m+1}=z$. We define $\sigma^{\prime}$ as follows:

$$
\sigma^{\prime}(i)=\left\{\begin{array}{lr}
\sigma(i), \quad \forall i \in\{1,2, \ldots, m\} \\
m+1, \quad i=m+1 .
\end{array}\right.
$$

It is clear that $\sigma^{\prime}$ is one to one and onto. Thus for any $r, s \in S$

$$
\left(\prod_{i=1}^{m+1} z_{i}\right)(r)>0 \text { and }\left(\prod_{i=1}^{m+1} z_{\sigma^{\prime}(i)}\right)(s)>0 \text {. }
$$

Hence $\sigma^{\prime}$ is a permutation of $\mathbb{S}^{m+1}$ such that $\sigma^{\prime}(i)=i$ if $z_{i} \notin A_{n}^{\alpha}(S)$. Therefore, $x z \overline{\overline{\xi_{n}^{\alpha}}} y z$. Now, if $x \xi_{n}^{* \alpha} y$, then there exists $k \in \mathbb{N}$ and $u_{0}=x, u_{1}, \ldots, u_{k}=y \in S$ such that $u_{0}=$ $x \xi_{n}^{\alpha} u_{1} \xi_{n}^{\alpha} u_{2} \xi_{n}^{\alpha} \ldots \xi_{n}^{\alpha} u_{k}=y$. By the above result we have $u_{0} z=x z \overline{\overline{\xi_{n}^{\alpha}}} u_{1} z \overline{\overline{\xi_{n}^{\alpha}}} u_{2} z \overline{\overline{\xi_{n}^{\alpha}}} \ldots \overline{\overline{\xi_{n}^{\alpha}}} u_{k} z=y z$ and so $x z \overline{\overline{\xi_{n}^{\alpha}}} y z$. By the similar way, we can show that $z x \overline{\overline{\xi_{n}^{\alpha}}} z y$. Therefore, $\xi_{n}^{* \alpha}$ is a fuzzy strongly regular relation on $S$.

Proposition 3.5. For any integer $n$ we have $\xi_{n+1}^{* \alpha} \subseteq \xi_{n}^{* \alpha}$.

Proof. Let $x \xi_{n+1}^{\alpha} y$. Then there exist $m \in \mathbb{N},\left(z_{1}, \ldots, z_{m}\right) \in S^{m}$ and $\delta \in \mathbb{S}_{m}$ with $\delta(i)=i$ if $z_{i} \notin A_{n+1}^{\alpha}(S)$ such that $\left(\prod_{i=1}^{m} z_{i}\right)(x)>0$ and $\left(\prod_{i=1}^{m} z_{\delta(i)}\right)(y)>0$. Now, for $\left(z_{1}, \ldots, z_{m}\right) \in S^{m}$ and $\delta \in$ $\mathbb{S}_{m}$ with $\delta(i)=i$ if $z_{i} \notin A_{n}^{\alpha}(S)$ we have $z_{i} \notin A_{n+1}^{\alpha}(S)$ (since $\left.A_{n+1}^{\alpha}(S) \subseteq A_{n}^{\alpha}(S)\right)$ and so $\left(\prod_{i=1}^{m} z_{i}\right)(x)>$ 0 and $\left(\prod_{i=1}^{m} z_{\delta(i)}\right)(y)>0$. Therefore, $x \xi_{n}^{\alpha} y$. 
Proposition 3.6. For any integer $n$ we have $\lambda^{*} \subseteq \xi_{n}^{* \alpha} \subseteq \epsilon^{*}$. In particular, if $S$ is a commutative $F H G$, then $\epsilon^{*}=\xi_{n}^{* \alpha}=\lambda^{*}$.

Proof. It is clear that $\lambda^{*} \subseteq \xi_{n}^{* \alpha} \subseteq \epsilon^{*}$. It is enough to show that if $S$ is commutative, then $\lambda^{*}=\xi_{n}^{* \alpha}=\epsilon^{*}$. For this, let $a \xi_{n}^{* \alpha} b$. Then there exists an integer $m,\left(x_{1}, x_{2}, \ldots, x_{m}\right) \in S^{m}$ and $\varrho \in \mathbb{S}^{m}$ with $\varrho(i)=i$ if $x_{i} \notin A_{n}^{\alpha}(S)$ such that $\left(x_{1} \circ \ldots \circ x_{m}\right)(a)>0$ and $\left(x_{\sigma_{1}} \circ \ldots \circ x_{\sigma_{m}}\right)(b)>0$. For any $i$ since $S$ is commutative, we conclude that each element $x_{\varrho(i)}$ can commute with others and so $\lambda^{*}=\xi_{n}^{* \alpha}=\epsilon^{*}$.

Example 3.7. Let $S$ be an FHG as Example [.?. Then it is routine to verify that $\rho$ is a fuzzy strongly regular relation $[\Pi]$.

Now, we are ready to state one of our main results of this section.

Theorem 3.8. $S / \xi_{n}^{* \alpha}$ is an $\alpha$-solvable group of length at most $n+1$.

Proof. Let $\varphi$ be a fuzzy strongly regular relation on $S$. Then we show that for any integer $k$

$$
\left.D_{k}^{\alpha}(S / \varphi)\right)=\left\langle\bar{t} \mid t \in A_{k}^{\alpha}(S)\right\rangle .
$$

We proceed by induction on $k$. Put $G=S / \varphi$. Since $G=\langle\bar{t} \mid t \in S\rangle$ the case $k=0$ is clear. Now, suppose that $\bar{a} \in\left\langle\bar{t} \mid t \in A_{k+1}^{\alpha}(S)\right\rangle$, then there exists $t \in A_{k+1}^{\alpha}(S)$ such that $\bar{a}=\bar{t}$. By Definition B.], there exist $r_{1} \in S$ and $x, y \in A_{k}^{\alpha}(S)$ such that $(x y)\left(r_{1}\right)>0$ and $\left(t \bullet y^{\alpha} x\right)\left(r_{1}\right)>0$. It follows from Theorem $\left[2.4\right.$ that $\bar{x} \oplus \bar{y}=\overline{r_{1}}$ and $\bar{t} \oplus \overline{y^{\alpha}} \oplus \bar{x}=\overline{r_{1}}=\bar{x} \oplus \bar{y}$. So $\bar{t}=[\bar{x}, \bar{y}] \bar{\alpha}$. The hypotheses of induction implies that $\bar{a}=\bar{t} \in D_{k+1}^{\alpha}(G)$.

Conversely, let $\bar{a} \in D_{k+1}^{\alpha}(G)$. Then there exist $\bar{x}, \bar{y} \in D_{k}^{\alpha}(G)$ such that $\bar{a}=[\bar{x}, \bar{y}]_{\bar{\alpha}}$. So by hypotheses of induction we have $\bar{x}=\bar{u}$ and $\bar{y}=\bar{v}$, where $u, v \in A_{k}^{\alpha}(S)$. As $u v$ is a nonzero fuzzy subset of $S$ so there exists $c \in S$ such that $(u v)(c)>0$. By Definition [2.], we have $1=\chi_{S}(c)=(S u)(c)=\bigvee_{r \in S}(r u)(c)$ and so there exists $r \in S$ such that $(r u)(c)>0$. Moreover, $1=\chi_{S}(r)=\left(S v^{\alpha}\right)(r)=\bigvee_{t \in S}\left(t v^{\alpha}\right)(r)$. Hence, by Definition [2.] we have:

$$
\left(t \bullet v^{\alpha} u\right)(c)=\left(t v^{\alpha} \bullet u\right)(c)=\bigvee_{p}\left(\left(t v^{\alpha}\right)(p) \wedge(p u)(c)\right) \geq\left(t v^{\alpha}\right)(r) \wedge(r u)(c)>0
$$

Thus $(u v)(c)>0$ and $\left(t \bullet v^{\alpha} u\right)(c)>0$. So $t \in A_{k+1}^{\alpha}(S)$. It follows from Theorem [2.4, that $\bar{u} \oplus \bar{v}=\bar{c}=\bar{t} \oplus \overline{v^{\alpha}} \oplus \bar{u}$, and so $\bar{t}=[\bar{u}, \bar{v}]_{\bar{\alpha}}=[\bar{x}, \bar{y}]_{\bar{\alpha}}=\bar{a}$. Therefore, $\bar{a}=\bar{t} \in\left\langle\bar{t} ; t \in A_{k+1}^{\alpha}(S)\right\rangle$ i.e $D_{k+1}^{\alpha}(S / \varphi)=\left\langle\bar{t} \mid t \in A_{k+1}^{\alpha}(S)\right\rangle$. Consequently, $D_{n}^{\alpha}\left(S / \xi_{n}^{* \alpha}\right)$ is an abelian group and $D_{n+1}^{\alpha}\left(S / \xi_{n}^{* \alpha}\right)=$ $\{e\}$.

In the following, we introduce the smallest fuzzy strongly regular relation $\xi^{* \alpha}$ on a finite FHG $S$ such that $S / \xi^{* \alpha}$ is an $\alpha$-solvable group.

Theorem 3.9. The fuzzy relation $\xi^{* \alpha}=\bigcap_{n \geq 1} \xi_{n}^{* \alpha}$ is the smallest fuzzy strongly regular relation on a finite FHG $S$ such that $S / \xi^{* \alpha}$ is an $\alpha$-solvable. In particular, $\xi^{* \alpha}$ is an $\alpha$-solvable fundamental relation.

Proof. First, we show that $\xi^{* \alpha}$ is a fuzzy strongly regular relation on $S$ such that $S / \xi^{* \alpha}$ is $\alpha$ solvable. By $\xi^{* \alpha}=\bigcap_{n \geq 1} \xi_{n}^{* \alpha}$ and Theorem [3.4, it is easy to see that $\xi^{* \alpha}$ is a fuzzy strongly regular 
relation on $S$. Since $S$ is finite, Proposition 3.5 implies that there exists an integer $k$ such that $\xi_{k+1}^{* \alpha}=\xi_{k}^{* \alpha}$. Thus, for some $m \xi^{* \alpha}=\xi_{m}^{* \alpha}$ and so by Theorem [3.8, $S / \xi^{* \alpha}$ is $\alpha$-solvable.

Now, we prove $\xi^{* \alpha}$ is the smallest relation with this property. Suppose $\rho$ is a fuzzy strongly regular relation on $S$ such that $K=S / \rho$ is $\alpha$-solvable of class $c$. We show that $\xi^{* \alpha} \subseteq \rho$. For this, let $x, y \in S$ and $x \xi^{\alpha} y$, where $\xi^{\alpha}=\bigcap_{n \geq 1} \xi_{n}^{\alpha}$. Then there exists integers $n$ and $m$ such that $x \xi_{m, n}^{\alpha} y$ and so there exist $\left(z_{1}, \ldots, z_{m}\right) \in S^{m}$ and $\delta \in \mathbb{S}_{m}$ with $\delta(i)=i$ if $z_{i} \notin A_{n}^{\alpha}(S)$ such that $\left(\prod_{i=1}^{m} z_{i}\right)(x)>0$ and $\left(\prod_{i=1}^{m} z_{\delta(i)}\right)(y)>0$. Thus by Theorem [2.4, we get

$$
\bar{x}=\prod_{i=1}^{m} \overline{z_{i}} \text { and } \bar{y}=\prod_{i=1}^{m} \overline{z_{\delta(i)}} .
$$

By the proof of Theorem B.8, we have

$$
D_{c}(S / \rho)=\left\langle\bar{t} \mid t \in A_{c}^{\alpha}(S)\right\rangle=\{\bar{e}\} .
$$

And so for any $z_{i} \in A_{c}^{\alpha}(S)$ we get $\overline{z_{i}}=\bar{e}$. Hence, $\bar{x}=\bar{y}$. Therefore, $x \rho y$ as required. Now, $\xi^{* \alpha} \subseteq \rho$, because, let $z, t \in S$ and $z \xi^{* \alpha} t$. Then for some integer $n, z \xi_{n}^{* \alpha} t$ and so there exist $z_{0}, z_{1}, \ldots, z_{k} \in S$ $(k \in \mathbb{N})$ such that $\left(z=z_{0}\right) \xi_{n}^{* \alpha} z_{1} \xi_{n}^{* \alpha} \ldots \xi_{n}^{* \alpha}\left(z_{k}=t\right)$. So we have $\left(z=z_{0}\right) \rho z_{1} \rho \ldots \rho\left(z_{k}=t\right)$. Hence, $\xi^{* \alpha} \subseteq \rho$. Therefore, $\xi^{* \alpha}$ is the smallest relation such that $S / \xi^{* \alpha}$ is an $\alpha$-solvable group.

Example 3.10. Let $S$ be an FHG as Example [3.9. Then, by Proposition [., w, we have $\epsilon^{*}=\xi_{n}^{* \alpha}$ and so $S / \xi_{n}^{* \alpha}=S / \epsilon * \cong S$. Therefore, it follows from Theorem 3.8 that $S$ is an $\alpha$-solvable group.

Example 3.11. Let $\alpha$ be the identity isomorphism and $S=\{a, b, c\}$. Consider fuzzy hyperoperation" $\circ "$ on $S$ as follows:

$(a \circ a)(a)=(b \circ b)(a)=(c \circ c)(a)=0.5, \quad(a \circ b)(b)=(b \circ a)(b)=(b \circ c)(b)=(c \circ b)(b)=$ $0.1,(a \circ c)(c)=(b \circ b)(c)=(c \circ a)(c)=0.7$, and $(a \circ a)(b)=(a \circ a)(c)=(a \circ b)(a)=(a \circ b)(c)=$ $(a \circ c)(a)=(a \circ c)(b)=(b \circ a)(a)=(b \circ a)(c)=(b \circ b)(b)=(b \circ c)(a)=(b \circ c)(c)=(c \circ a)(a)=$ $(c \circ a)(b)=(c \circ b)(a)=(c \circ b)(c)=(c \circ c)(b)=(c \circ c)(c)=0$.

Let $\rho_{1}=\{(a, a),(b, b),(c, c)\}$. It is clear that $\rho_{1}$ is the smallest fuzzy strongly regular relation.

\section{$4 \xi^{\alpha}$-part of an FHG}

In this section, we use the concept of an $\xi^{\alpha}$-part of an FHG to make a transitive fuzzy relation $\xi^{\alpha}$ on an FHG.

Definition 4.1. Let $X$ be a non-empty subset of $S$. Then $X$ is called an $\xi^{\alpha}$-part of $S$ if for any $m \in \mathbb{N},\left(z_{1}, \ldots, z_{m}\right) \in S^{m}$ and $\sigma \in \mathbb{S}_{m}$ with $\sigma(i)=i$ if $z_{i} \notin \bigcup_{n \geq 1} A_{n}^{\alpha}(S)$, then

$$
\text { there exists } x \in X \text { such that }\left(\prod_{i=1}^{m} z_{i}\right)(x)>0 \text { implies for all } y \in S \backslash X,\left(\prod_{i=1}^{m} z_{\sigma(i)}\right)(y)=0 \text {. }
$$

Theorem 4.2. Let $X$ be a non-empty subset of $S$. Then for any $x, y \in S$ the following conditions are equivalent: 
(i) $X$ is an $\xi^{\alpha}$-part of $S$,

(ii) If $x \in X$ and $x \xi^{\alpha} y$, then $y \in X$,

(iii) If $x \in X$ and $x \xi^{* \alpha} y$, then $y \in X$.

Proof. $(i) \Rightarrow(i i)$ For $x, y \in S$ if $x \in X$ and $x \xi^{\alpha} y$, then there exist $n, m \in \mathbb{N}$ such that $x \xi_{m, n}^{\alpha} y$ and so there exist $\left(z_{1}, \ldots, z_{m}\right) \in S^{m}$ and $\sigma \in \mathbb{S}_{m}$ with $\sigma(i)=i$ if $z_{i} \notin \bigcup_{n \geq 1} A_{n}^{\alpha}(S)$ such that $\left(\prod_{i=1}^{m} z_{i}\right)(x)>0$ and $\left(\prod_{i=1}^{m} z_{\sigma(i)}\right)(y)>0$. As $X$ is an $\xi^{\alpha}$-part of $S$ and $\left(\prod_{i=1}^{m} z_{i}\right)(x)>0$ if $y \notin X$ we have $\left(\prod_{i=1}^{m} z_{\sigma(i)}\right)(y)=0$, a contradiction. Therefore, $y \in X$.

$($ ii $) \Rightarrow($ iii $)$ Let $x, y \in S, x \in X$, and $x \xi^{* \alpha} y$. Then there is an integer $m$ and $\left(z_{0}, \ldots, z_{m}\right) \in S^{m}$ such that $x=z_{0} \xi^{\alpha} z_{1} \xi^{\alpha} \ldots \xi^{\alpha} z_{m}=y$. Applying (ii) $m$ times, we have $y \in X$.

$(i i i) \Rightarrow(i)$ For $\left(z_{1}, \ldots, z_{m}\right) \in \mathbb{S}^{m}$ and $\sigma \in S_{m}$ with $\sigma(i)=i$ if $z_{i} \notin \bigcup_{n \geq 1} A_{n}^{\alpha}(S)$, let $x \in X$ and $\left(\prod_{i=1}^{m} z_{i}\right)(x)>0$. If $y \notin X$, then $\left(\prod_{i=1}^{m} z_{\sigma(i)}\right)(y)>0$. It follows that $x \xi_{n} y$ and so $x \xi y$. Hence, (iii) implies that $y \in X$, a contradiction and so $\left(\prod_{i=1} z_{\sigma(i)}\right)(y)=0$, i.e $X$ is an $\xi^{\alpha}$-part of $S$.

Example 4.3. Let $X=\{a, c\}$ be as Example [3.7. Then by Theorem G.9 and Proposition [3.6, $X$ is an $\xi^{\alpha}$-part of $S$.

Theorem 4.4. For any $a \in S, \xi^{\alpha}(a)$ is an $\xi^{\alpha}$-part of $S$ if and only if $\xi^{\alpha}$ is transitive.

Proof. $(\Leftarrow)$ Let $x, y \in S, z \in \xi^{\alpha}(x)$ and $z \xi^{\alpha} y$. Since $\xi^{\alpha}$ is transitive, we have $y \in \xi^{\alpha}(x)$. So, by Theorem 4.2, $\xi^{\alpha}(x)$ is an $\xi^{\alpha}$-part of $S$.

$(\Rightarrow)$ Suppose that $x \xi^{* \alpha} y$. Then there exists an integer $k$ and $\left(z_{1}, \ldots, z_{k}\right) \in S^{k}$ such that

$$
x=z_{0} \xi^{\alpha} z_{1} \xi^{\alpha} \ldots \xi^{\alpha} z_{k}=y
$$

thus, $z_{i} \in \xi^{\alpha}\left(z_{i-1}\right)$. Since $\xi^{\alpha}\left(z_{i}\right)$ is an $\xi^{\alpha}$-part $(0 \leq i \leq k)$ it follows that $y \in \xi^{\alpha}(x)$ by Theorem 4.2, i.e $x \xi^{\alpha} y$ and so $\xi^{* \alpha}=\xi^{\alpha}$.

Definition 4.5. Let $A$ be a non-empty subset of $S$. We define $K(A)$ and $W(A)$ as follows:

1) $K(A)=\bigcap\left\{B: A \subseteq B\right.$ and $B$ is an $\xi^{\alpha}$ - part of $\left.S\right\}$. We use $K(a)$ for $K(\{a\})$,

2) $W(A)=\bigcup_{n \geq 1} W_{n}(A)$, where $W_{1}(A)=A$ and for $n \geq 1$,

$W_{n+1}(A)=\left\{x \in S \mid \exists m \in \mathbb{N}\right.$ and $\exists\left(z_{1}, \ldots, z_{m}\right) \in S^{m}$ such that for some $a \in W_{n}(A)$ we have

$$
\left.\left(\prod_{i=1}^{m} z_{i}\right)(x)>0 \text { and } \exists \sigma \in \mathbb{S}_{m} \text { with } \sigma(i)=i \text { if } z_{i} \notin \bigcup_{s \geq 1} A_{s}^{\alpha}(S) \text { such that }\left(\prod_{i=1}^{m} z_{\sigma(i)}\right)(a)>0\right\} \text {. }
$$

Example 4.6. Let $A=\{a, c\}$ be as Example [3.7. Since $X$ is an $\xi^{\alpha}$-part of $S$ we have $K(A)=A$. 
Theorem 4.7. The following statements hold:

(1) $W(A)=K(A)$,

(2) $K(A)=\bigcup_{a \in A} K(a)$

(3) $W_{n}\left(W_{2}(z)\right)=W_{n+1}(z)$, for $n \geq 2$ and $z \in S$.

Proof. (1) We show that $W(A)$ is an $\xi^{\alpha}$-part. Let $a \in W(A),\left(\prod_{i=1}^{m} z_{i}\right)(a)>0$ and $\sigma \in \mathbb{S}_{m}$ with $\sigma(i)=i$, if $z_{i} \notin \bigcup_{s \geq 1} A_{s}^{\alpha}(S)$. Then there exists an integer $n$ such that $a \in W_{n}(A)$. If $t \notin W(A)$ and $\left(\prod_{i=1} z_{\sigma(i)}\right)(t)>0$, then $t \in W_{n+1}(A)$ and so $t \in W(A)$, a contradiction. Therefore, $\left(\prod_{i=1}^{m} z_{\sigma(i)}\right)(t)=0$ and $W(A)$ is an $\xi^{\alpha}$-part.

Now, it is enough to prove that if $B$ is an $\xi^{\alpha}$-part and $A \subseteq B$, then for any $n, W_{n}(A) \subseteq B$ i.e $W(A)$ is the smallest $\xi^{\alpha}$-part of $S$ which contains $A$. We use induction on $n$. Since $W_{1}(A)=A \subseteq B$, the case $n=1$ is clear. Let $W_{n}(A) \subseteq B$ and $z \in W_{n+1}(A)$. Then there exists an integer $m$ and $\left(z_{1}, \ldots, z_{m}\right) \in S^{m}$ and $\sigma \in \mathbb{S}_{m}$ with $\sigma(i)=i$ if $z_{i} \notin \bigcup_{s \geq 1} A_{s}^{\alpha}(S)$ and $t \in W_{n}(A)$ such that $\left(\prod_{i=1}^{m} z_{\sigma(i)}\right)(t)>0$ and $\left(\prod_{i=1}^{m} z_{i}\right)(z)>0$. Since $W_{n}(A) \subseteq B$ we have $t \in B$. Moreover, if $z \notin B$ as $B$ is $\xi^{\alpha}$-part, then $\left(\prod_{i=1}^{m} z_{i}\right)(z)=0$, a contradiction, and so $z \in B$ and the result holds.

(2) We know that for any $a \in A, K(a) \subseteq K(A)$. We use induction on $n$ to prove that $W_{n}(A)=\bigcup_{a \in A} W_{n}(a)$. It follows from $(1)$ that $K(A)=\bigcup_{n \geq 1} W_{n}(A)$ and $W_{1}(A)=A=\bigcup_{a \in A}\{a\}$. Suppose that it is true for $n$ and $z \in W_{n+1}(A)$. Then there exists an integer $m$ and $\left(z_{1}, \ldots, z_{m}\right) \in$ $S^{m}$ such that $\left(\prod_{i=1}^{m} z_{i}\right)(z)>0$ and there exists $\sigma \in \mathbb{S}_{m}$ with $\sigma(i)=i$ if $z_{i} \notin \bigcup_{s \geq 1} A_{s}^{\alpha}(S)$ such that for some $a \in W_{n}(A),\left(\prod_{i=1}^{m} z_{\sigma(i)}\right)(a)>0$. By the hypotheses of induction we have $W_{n}(A)=\bigcup_{b \in A} W_{n}(b)$ and so $a \in \bigcup_{b \in A} W_{n}(b)$. Therefore, for some $b \in A, a \in W_{n}(b)$. Hence, $z \in W_{n+1}(b)$ i.e $W_{n+1}(A) \subseteq$ $\bigcup_{b \in A} W_{n+1}(b)$. Since for any $a \in A, K(a) \subseteq K(A)$ we obtain $K(A)=\bigcup_{n} W_{n}(A) \subseteq \bigcup_{n} \bigcup_{a} W_{n}(a)=$ $\bigcup_{a \in A} K(a) \subseteq K(A)$ Therefore, $K(A)=\bigcup_{a \in A} K(a)$.

(3) We proceed by induction on $n$. For $n=2$ we have

$$
\begin{aligned}
W_{2}\left(W_{2}(x)\right)= & \left\{z \mid \exists q \in \mathbb{N}, \exists\left(a_{1}, \ldots, a_{q}\right) \in S^{q} \text { and } \sigma \in \mathbb{S}_{q} \text { with } \sigma(i)=i \text { if } z_{i} \notin \bigcup_{s \geq 1} A_{s}^{\alpha}(S)\right. \text { such that } \\
& \left.\left(\prod_{i=1}^{q} a_{i}\right)(z)>0 \text { and for some } y \in W_{2}(x),\left(\prod_{i=1}^{q} a_{\sigma(i)}\right)(y)>0\right\}=W_{3}(x) .
\end{aligned}
$$


Suppose $W_{n}\left(W_{2}(x)\right)=W_{n+1}(x)$. Then

$$
\begin{aligned}
W_{n+1}\left(W_{2}(x)\right)= & \left\{z \in S \mid \exists q \in \mathbb{N},\left(a_{1}, \ldots, a_{q}\right) \in S^{q} \text { and } \sigma \in \mathbb{S}_{q} \text { with } \sigma(i)=i \text { if } z_{i} \notin \bigcup_{s \geq 1} A_{s}^{\alpha}(S),\right. \\
& \left.\left.t \in W_{n}\left(W_{2}(x)\right) \text { such that }\left(\prod_{i=1}^{q} a_{i}\right)(z)>0 \text { and } \prod_{i=1}^{q} a_{\sigma(i)}\right)(t)>0\right\} \\
= & W_{n+2}(x) .
\end{aligned}
$$

This completes the proof.

Theorem 4.8. Let $x, y \in S$. Then the following relation is an equivalence relation on $S$ :

$x W y$ if and only if $x \in W(\{y\})$.

Proof. The relation $W$ is reflexive, since Theorem 4.7 and Definition 4.5 , imply that $W\{x\}=K\{x\}$ and $x \in W\{x\}$ i.e $x W x$. Also, $W$ is transitive, since for $x, y, z \in S$ let $x W y$ and $y W z$. Therefore, Theorem 4.7, implies $x \in K(y)$ and $y \in K(z)$. For any $P, \xi^{\alpha}$-part of $S$ which contains $z$, we have $K(z) \subseteq P$ and so $y \in P$. Then $K(y) \subseteq P$ and so $x \in P$. Thus for any $P$ we have $x \in P$ and $K(z)$ is an $\xi^{\alpha}$-part of $S$ which contains $z$, so $x \in K(z)$. Therefore, by Theorem 4.7, $x W z$ and so $W$ is transitive. $W$ is symmetric. For this first by induction on $n$ we prove that $x \in W_{n}(y)$ if and only if $y \in W_{n}(x)$. For $n=2$ it is clear. Suppose $x \in W_{n+1}(y)$, then there exists an integer $q \geq 1,\left(a_{1}, \ldots, a_{q}\right) \in S^{q}$ and $\sigma \in \mathbb{S}_{q}$ with $\sigma(i)=i$ if $a_{i} \notin \bigcup_{s \geq 1} A_{s}^{\alpha}(S)$ and $t \in$ $W_{n}(y)$ such that $\left(\prod_{i=1}^{q} a_{i}\right)(x)>0$ and $\left(\prod_{i=1}^{q} a_{\sigma(i)}\right)(t)>0$. It follows that $t \in W_{2}(x)$. By hypotheses of induction we have $y \in W_{n}(t)$. Therefore, by Theorem $4.7(3)$, we have $y \in W_{n}\left(W_{2}(x)\right)=W_{n+1}(x)$.

Example 4.9. Let $\rho=\{(a, a),(b, b),(c, c),(a, c),(c, a)\}$ and $\pi: S \rightarrow S / \rho$ defined by $\pi(x)=\bar{x}$ for all $x \in S$ be the canonical homomorphism. We know that $\rho$ is a fuzzy strongly regular relation so by Theorem [2., $S / \rho$ is a group. Moreover, $S / \rho=\{\bar{a}, \bar{b}\}$ and $\bar{a}=\{a, c\}$ is the identity element of $S / \rho$. Also,

$$
\omega_{S}=\operatorname{Ker}(\pi)=\{x \mid \bar{x}=\bar{a}\}=\{a, c\} .
$$

By Example [.马, $\{a, c\}$ is a $\rho$-part of $S$ i.e $\omega_{S}$ is a $\rho$-part of $S$.

Let $M$ be a non-empty subset of $S$. We Know that $\left(M \omega_{S}\right)(r)=\bigvee_{x \in \omega_{S}, m \in M}(m \circ x)(r)$.

Lemma 4.10. Assume that $M$ is a non-empty subset of $S$. Then we have (i) $\pi^{-1}(\pi(M))=\left\{x \in S:\left(\omega_{S} M\right)(x)>0\right\}=\left\{x \in S:\left(M \omega_{S}\right)(x)>0\right\}$;

(ii) If $M$ is an $\xi^{\alpha}$-part of $S$, then $\pi^{-1}(\pi(M))=M$.

Proof. (i) Let $x \in S, t \in \omega_{S}$ and $y \in M$ such that $(t y)(x)>0$. Then by Theorem [2.4, $\pi(x)=$ $\pi(t) \oplus \pi(y)=1_{S / \xi^{* \alpha}} \oplus \pi(y)=\pi(y)$ and so $x \in \pi^{-1}(\pi(y)) \subset \pi^{-1}(\pi(M))$.

Conversely, for any $x \in \pi^{-1}(\pi(M))$, there exists $b \in M$ such that $\pi(x)=\pi(b)$. For $a \in S$ we have $a S=\chi_{S}$ and so $(a b)(x)>0$. Since by Theorem [2.4, $\pi(b)=\pi(x)=\pi(a) \oplus \pi(b)$ we have $\pi(a)=1_{S / \xi^{* \alpha}}$. So $a \in \pi^{-1}\left(1_{S / \xi^{* \alpha}}\right)=\omega_{S}$. Therefore, $\left(\omega_{S} M\right)(x)>0$. 
By the similar way, we can prove that $\pi^{-1}(\pi(M))=\left\{x \in S:\left(M \omega_{S}\right)(x)>0\right\}$.

(ii) It is clear that $M \subseteq \pi^{-1}(\pi(M))$. If $x \in \pi^{-1}(\pi(M))$, then there exists $b \in M$ such that $\pi(x)=\pi(b)$ i.e $\xi^{* \alpha}(x)=\xi^{* \alpha}(b)$. Therefore, $x \in M$ by Theorem $4.2\left(\right.$ iii) and $M$ is $\xi^{\alpha}$-part.

Theorem 4.11. For all $a, b \in S, a W b$ if and only if $a \xi^{* \alpha} b$.

Proof. $(\Leftarrow)$ Let $a \xi^{* \alpha} b$. Then there exist integer $n, m$ such that $a \xi_{m, n}^{\alpha} b$. So for any $\left(z_{1}, \ldots, z_{m}\right) \in S^{m}$ and $\sigma \in \mathbb{S}_{m}$ with $\sigma(i)=i$ if $z_{i} \notin \bigcup_{n \geq 1} A_{n}^{\alpha}(S)$ we have $\left(\prod_{i=1}^{q} a_{i}\right)(a)>0$ and $\left(\prod_{i=1}^{q} a_{\sigma(i)}\right)(b)>0$ and so $a \in W_{2}(b)$. Thus, by Definition $4.5, a W b$ and $\xi^{* \alpha} \subset W$.

$(\Rightarrow)$ If $x W y$, then there exists $n \in \mathbb{N}$ such that $x \in W_{n}(y)$. So for any integer $m,\left(z_{1}, \ldots, z_{m}\right) \in S^{m}$ and $\sigma \in \mathbb{S}_{m}$ with $\sigma(i)=i$ if $z_{i} \notin \bigcup_{n \geq 1} A_{n}^{\alpha}(S)$ we have $\left(\prod_{i=1}^{q} a_{i}\right)(x)>0$ and for some $x_{1} \in W_{n-1}(y)$ we have $\left(\prod_{i=1}^{q} a_{\sigma(i)}\right)\left(x_{1}\right)>0$. Thus, $x \zeta_{n}^{\alpha} x_{1}$. Continuing this method there exist $\exists x_{2}, \ldots, x_{n-1} \in S$ such that $x_{i} \in W_{n-i}(y)$ and $x_{i-1} \xi_{n}^{\alpha} x_{i}$. Then $\left(x=x_{0}\right) \xi_{n}^{\alpha} x_{1} \xi_{n}^{\alpha} \ldots \xi_{n}^{\alpha}\left(x_{n-1}=y\right)$. Therefore, $W \subseteq \zeta^{* \alpha}$.

Theorem 4.12. $\omega_{S}$ is a fuzzy subhypergroup of $S$ which is also an $\xi^{\alpha}$-part of $S$.

Proof. It is clear that $\omega_{S} \subseteq S$ and so for any $a, b, c \in \omega_{S},(a b) \bullet c=a \bullet(b c)$. Let $x, y \in \omega_{S}$. Then $S y=\chi_{S}$ implies that there exists $u \in S$ such that $(u y)(x)>0$. By Theorem $\overline{L .4}, \bar{u} \oplus \bar{y}=\bar{x}$ and so $\bar{u}=\overline{1}$. i.e $u \in \omega_{S}$. Therefore, $\omega_{S} y=\chi_{\omega_{S}}$ and $\omega_{S}$ is a fuzzy subhypergroup of $S$. Now we prove that

$$
K(y)=\pi^{-1}(\pi(\{y\}))=\left\{x \in S:\left(\omega_{S} y\right)(x)>0\right\}=\omega_{S} .
$$

Let $y, z \in S$. Then

$$
\begin{aligned}
z \in \pi^{-1}(\pi(\{y\})) & \Longleftrightarrow \pi(z)=\pi(y) \\
& \Longleftrightarrow \xi^{* \alpha}(z)=\xi^{* \alpha}(y) \\
& \Longleftrightarrow z \xi^{* \alpha} y \\
& \Longleftrightarrow z \in \xi^{* \alpha}(y)=W(\{y\})=K(y) .
\end{aligned}
$$

Moreover, $y \in \omega_{S}$, we have $\left\{x \in S:\left(\omega_{S} y\right)(x)>0\right\}=\left\{x \in S:\left(\chi_{\omega_{S}}\right)(x)>0\right\}=\omega_{S}$. Therefore, $K(y)=\omega_{S}$ and so $\omega_{S}$ is an $\xi^{\alpha}$-part.

\section{Conclusions}

In this paper, we defined a new strongly regular relation on an FHG to get an $\alpha$-solvable group. Also, we introduced the concept of $\xi^{\alpha}$-part of a fuzzy hypergroup. Basically, we studied the relation between their fundamental relation and $\xi^{\alpha}$-parts of a given FHG. In addition, we can extend this work on $\alpha$-Engel groups ( $\alpha$-nilpotant groups). 


\section{References}

[1] R. Ameri, T. Nozari, Complete parts and fundamental relation on fuzzy hypersemigroups, Journal of Multiple-Valued Logic and Soft Computing, 19 (2011), 451-460.

[2] R. Barzegar, A. Erfanian, Nilpotency and solubility of groups relative to an automorphism, Caspian Journal of Mathematical Sciences, 4(2) (2015), 271-283.

[3] R.A. Borzooei, E. Mohammadzadeh, V. Fotea, On Engel fuzzy subpolygroups, New Mathematics and Natural Computation, 13(2) (2017), 165-206.

[4] P. Corsini , Prolegomena of hypergroup theory, Aviani Editore, Tricesimo, 1993.

[5] P. Corsini, I. Tofan, On fuzzy hypergroups, Pure Mathematics and Applications, 8 (1997), $29-37$.

[6] D, Freni, A new charactrization of the drived hypergroup via strungly regular equivalences, Communications in Algebra, 30(8) (2002), 3977-3989.

[7] V. Leoreanu-Fotea, B. Davvaz, Fuzzy hyperrings, Fuzzy Sets and Systems 160(2009), 23662378 .

[8] E. Mohammadzadeh, R.A. Borzooei, Y.B. Jun, Results on Engel fuzzy subgroup, Algebraic Structures and their Applications, 4(2) (2017), 1-14.

[9] E. Mohammadzadeh, R.A. Borzooei, Nilpotent fuzzy subgroups, Mathematics, 6(27) (2018), $1-12$.

[10] J.N. Mordeson, M.S. Malik, Fuzzy commutative algebra, Journal of Fuzzy Mathematics, 3 (1995), 1-15.

[11] T. Nozari, Commutative fundamental relation in fuzzy hypersemigroups, Italian Journal of Pure and Applied Mathematics, 36 (2016), 455-464.

[12] A. Rosenfeld, Fuzzy groups, Journal of Mathematical Analysis and Applications, 35 (1971), $512-517$.

[13] M.K. Sen, R. Ameri, G. Chowdhury, Fuzzy hypersemigroups, Soft Computing, 12 (2008), 891-900.

[14] L.A. Zadeh, Fuzzy sets, Information Control, 8 (1965), 338-353. 